warnings and legislation, the negative consequences are invariably minimised. Again, I choose not to underestimate the intelligence of the majority of individuals who get the balance right, and also the plight and intelligence of those so-called criminals who eventually get it right.

I will never forget being exposed to young, burned-out addicts in a central district of Zurich. Some were sound asleep, no doubt needled into unconsciousness. Nearby was a caravan that supplied sterile needles to avoid, among other complications, HIV contamination. On hand were health care workers who could deal with emergencies and for some the possibility of rehabilitation. No drug dealers were to be seen. It is illegal to deal in drugs in Switzerland. And so it should be. What struck me about this approach, and to which I was so foreign, was that it was a realistic attempt to deal with a problem that affects a small percentage of the population. Although this is expensive, it costs less than the wars on drug cartels, other drugrelated crimes and criminals. In other words, if you're into drugs, do it where you can be seen and, if necessary, helped. I agree with the view that to commit a crime because of drug abuse is a criminal act, but to have a vice is not.

\section{Ian McCallum}

Cape Town

soitgoes@iafrica.com

1. Van Niekerk JP deV. Time to decriminalise drugs? S Afr Med J 2011;101(2):79-80.

\section{Health technology assessment in South Africa - future promise}

To the Editor: The scope of technologies for health extends from technologies that provide a direct benefit to health (such as molecular genetics, biological technologies, pharmaceuticals and medical devices) to those that support health system functions (such as telecommunications, information technologies, devices for environmental protection and food technologies). ${ }^{1}$ Appropriate technology in a health setting should be scientifically sound, adapted to local needs, acceptable to the community, maintained as far as possible by the people themselves in keeping with the principle of self-reliance, and capable of being applied with resources that the community and the country can afford. This requires development of well-defined policies and programmes delivered through a countrywide system incorporating the above concepts and based on a decentralised model such as the district health system in South Africa. ${ }^{2}$ Over the past two decades, the health technology (HT) situation in South Africa has been changing rapidly. ${ }^{3}$ However, the fragmented policy environment relating to these changes has led to unco-ordinated development of policies and guidelines. Against this background we assessed the current state of implementation of HT in South Africa and attempted to identify future challenges.

A qualitative study design involved interviewing a convenient sample of practitioners and experts from public hospitals, provincial departments of health and academic institutions $(N=32)$. We found that not every province in South Africa has developed its own HT policies, although development, procurement and use of health technology fall within provincial jurisdiction in terms of the National Health Act. ${ }^{2}$

Participants from provinces without HT policies believed that lack of clear policies and guidelines often resulted in haphazard procurement affecting service delivery in their health facilities. Challenges include inappropriate equipment, unavailability of consumables and spare parts, high maintenance costs and short lifespan. All suggested that establishment of dedicated agencies for HT at provincial and district levels would improve the current situation, provided their 
membership is made up of experts in the field. Consolidation of existing good practices and their relevance and effectiveness, particularly in a local setting, would be important enablers, whereas unavailability of trained manpower and lack of understanding of HT processes and their usefulness and impact on improvement of health care would be major barriers for future activities. ${ }^{4}$ They felt the need for health professionals, health managers from departments of health and academic institutions to work together for development of an enabling environment for use of efficient, effective and relevant HT in South Africa to make a greater impact.

We conclude that progress has been made in the past two decades in this area, but there is still a need for more co-ordinated effort to improve health outcomes. This requires a concerted effort from the departments of health and academic institutions.

\section{Debjani B Mueller}

Centre for Research on Health Economics, Social and Health Care Management (CREMS)

University Carlo Cattaneo (LIUC), and

Clinical Epidemiology Research and Training Centre (CERTC)

University of the Witwatersrand

Johannesburg

\section{Moreshnee Govender}

CERTC and School of Public Health

University of the Witwatersrand

\section{Debashis Basu}

CERTC and Department of Community Health

Charlotte Maxeke Johannesburg Academic Hospital and

University of the Witwatersrand

1. World Health Organization. The World Health Report 1998 - Life in the 21st Century: A Vision for All Geneva: WHO, 1998.
2. South African Government. National Health Act (Act 61 of 2003). Pretoria: Government Printer, 2004 3. Govender M, Letshokgohla ME, Basu D. Health technology assessment - a new initiative in South Africa. S Afr Med J 2010;100(6):334

4. Rajan A, Gutierrez-Ibarluzea I, Moharra M. Addressing issues in health technology assessment promotion: Motives, enablers, and barriers. Int J Technol Assess Health Care 2011;27(1):55-63.

\section{Launch of the Southern African Human Genome Programme}

To the Editor: The Southern African Human Genome Programme (SAHGP) is a ground-breaking national and regional initiative that aims to unlock the unique genetic character of southern African populations. Through generous support from the Department of Science and Technology (DST), the SAHGP was officially launched on 27 and 28 January 2011. Its vision is to improve quality of life by understanding human genetic diversity, to be achieved via the following objectives:

1. developing capacity for genomic research in southern Africa

2. establishing a sustainable resource for genomic research (including a regional sample repository and database)

3. translating the information and knowledge into improvements in human health.

The SAHGP includes scientists, medical practitioners, biostatisticians, ethicists, government representatives, lawyers and industry representatives, and is managed and led by southern Africans (emphasising the critical importance of sovereignty); its outputs will benefit southern African populations. The SAHGP will therefore focus specifically on southern African genomes; one of its most important objectives is to build local capacity for genomerelated research to ensure that this programme remains true to its objectives of being Africo-centric and is sustainable.

Evidence suggests that humans evolved in Africa; consequently, the African gene pool is likely to hold import information regarding the 